\title{
Diagnostic and Therapeutic Strategy for Acute Pulmonary Thromboembolism
}

\author{
Masahito Sakuma ${ }^{1}$, Mashio Nakamura ${ }^{2}$, Norifumi Nakanishi ${ }^{3}$, Yoshiyuki Miyahara ${ }^{4}$, \\ Nobuhiro Tanabe ${ }^{5}$, Norikazu Yamada ${ }^{2}$, Shigefumi Fukui ${ }^{1}$, Huan Wang ${ }^{1}$, Takayuki Kuriyama ${ }^{5}$, \\ Takeyoshi Kunieda $^{6}$, Tsuneaki Sugimoto ${ }^{7}$, Takeshi Nakano ${ }^{2}$ and Kunio Shirato ${ }^{1}$
}

\begin{abstract}
Background The diagnostic and therapeutic strategy for acute pulmonary thromboembolism (APTE) was published by the Japanese Circulation Society. But in Japan, there has been no report on how to improve the pre-test probability in APTE-suspected cases, to determine a practically available diagnostic strategy, nor has been a report that compares diagnostic methods and therapies for APTE by decision analysis.

Methods and Results APTE was found in $66.7 \%$ before using diagnostic imaging techniques. Compared with the absence of APTE, prolonged immobilization, cancer, tachycardia, unilateral leg swelling and inverted $\mathrm{T}$-wave in $\mathrm{V}_{1-3}$ were found more often in the presence of APTE. The rate of obtaining the result on the day of ordering the examination test was $100 \%$ with arterial blood gas analysis, trans-thoracic echocardiography and computed tomography (CT), $78.2 \%$ in D-dimer, $85.5 \%$ in pulmonary angiography, and $54.5 \%$ in perfusion lung scan. Decision analysis showed that the highest expected utility was anticoagulant over 0.51 in pre-test probability, with CT between 0.13 and 0.51 .

Conclusions The pre-test probability of APTE has already been high before using specific diagnostic imaging techniques in Japan. Our results showed that the diagnostic strategy for APTE made by the Japanese Circulation Society was available in most hospitals in Japan.
\end{abstract}

Key words: pulmonary embolism, pre-test probability, computed tomography, D-dimer, pulmonary angiography

(DOI: 10.2169/internalmedicine.45.1732)

\section{Introduction}

The number of patients with acute pulmonary thromboembolism (APTE) has increased in recent years (1-3). One of the reasons is that the diagnostic power has been improving (4). Because the diagnostic power varies among hospitals, it is important to make a diagnostic strategy for APTE. A way to elevate the pre-test probability in patients suspected of APTE has been sought (5-7), and D-dimer, perfusion lung scan, and venous ultrasonography of the legs were determined to be central to the diagnosis of APTE in the guidelines of Western countries (8-10). These diagnostic strategies of Western countries could not be accepted completely in Japan because of some differences in the actual conditions in Japan. Therefore, an original diagnostic strategy for Japan has been sought. As for the therapeutic strategy, it has not been determined in which cases thrombolytic agents should be administered.

In the present study, we examined ways to improve the pre-test probability in APTE-suspected cases, and determine a practically available diagnostic strategy for use in Japan,

\footnotetext{
${ }^{1}$ Department of Cardiovascular Medicine, Tohoku University Graduate School of Medicine, Sendai, ${ }^{2}$ Department of Cardiology, Mie University Graduate School of Medicine, Tsu, ${ }^{3}$ Department of Internal Medicine, National Cardiovascular Center, Osaka, ${ }^{4}$ Second Department of Internal Medicine, Nagasaki University, Nagasaki, ${ }^{5}$ Department of Chest Medicine, Chiba University, Chiba, ${ }^{6}$ Department of Internal Medicine, Sumida Shuko-en Facility, Tokyo and ${ }^{7}$ Kanto Central Hospital, Tokyo

Received for publication January 17, 2006; Accepted for publication April 27, 2006

Correspondence to Masahito Sakuma, Department of Cardiovascular Medicine, Tohoku University Graduate School of Medicine, 1-1 Seiryomachi, Aoba-ku, Sendai 980-8574
} 
based on questionnaires about examinations employed in emergencies by members of the Japanese Society of Pulmonary Embolism Research (JaSPER). And we also examined the diagnostic and therapeutic strategy using decision analysis.

\section{Method}

The present study is made up of three parts.

1. Prospective registry of cases suspected of APTE. We enrolled prospectively cases suspected of APTE to determine the findings effective for diagnosing APTE, and the accuracy of diagnosis before making special imaging examinations. Data were collected for: 1) risk factors, 2) circumstances at onset, 3) symptoms, 4) signs, 5) findings of chest roentgenogram, electrocardiogram, and echocardiography, and level of D-dimer, 6) diagnostic judgment of the participating physician without special imaging examinations, and 7) final diagnosis.

2. Survey of the examination tests of APTE. We sent questionnaires to 100 institutions belonging to JaSPER in January 2004. The response rate was 55\%. Data were collected for: 1) the duration from ordering the examination (blood gas analysis, D-dimer, trans-thoracic echocardiography, perfusion lung scan, ventilation lung scan, pulmonary angiography, computed tomography (CT), magnetic resonance (MR) angiography, and trans-esophageal echocardiography) until getting the result of the examination, 2) type of D-dimer test, and 3) type of CT (single or multi detector-row CT).

3. Decision analysis. We performed decision analysis on the Japanese original diagnostic procedures for APTE using the results from the two examinations described above, with reference to the sensitivity and specificity of examination tests reported in the literature (11-14). The values we used are listed in Table 1 . We dealt with 10 events of morbidity as one event of mortality as in Perrier's report (11). The expected utility was calculated as 100 minus the sum of the utility loss, where utility loss = mortality $(\%)+$ modality $(\%) / 10$. The utility loss occurred from APTE itself, examination, and medication (see APPENDIX).

In the present study, we used two decision trees. One is shown in Fig. 1-(1), where we assessed the examination test and use of anticoagulants. The other is shown in Fig. 1-(2), where examination was limited to CT for simplification, and we examined the possibility of thrombolytic agents for APTE.

\section{Statistical analysis}

Statistical analysis was carried out using SPSS 12.0 (SPSS Inc., Chicago, Il, USA). All continuous variables were analyzed by Mann-Whitney test and expressed as the median and 25-75 percentiles. Quantitative data were analyzed by Mann-Whitney test. Categorical data were analyzed by chi-square statistics. All significant tests were two-tailed.
Table 1. Values Used in the Decision Analysis

\begin{tabular}{lr}
\hline Pulmonary Thromboembolism & 30 \\
Loss of Utility: Without Treatment & 8 \\
Loss of Utility: Anticoagulant & 86 \\
Computed Tomography & 93 \\
Sensitivity $* 1$ & 0 \\
Specificity $* 1$ & \\
Loss of Utility $* 2$ & 90 \\
PAG & 95 \\
Sensitivity & 0.5 \\
Specificity & 0.3 \\
Loss of Utility: Mortality Before Completing PAG & \\
Loss of Utility: Cases with Completing PAG & 96 \\
Plasma D-dimer & 47 \\
Sensitivity & \\
Specificity & \\
Anticoagulant & \\
Loss of Utility & \\
Anticoagulant + Thrombolysis & \\
Loss of Utility $* 3$ & \\
\hline
\end{tabular}

$* 1$ from ref. (12), $* 2$ estimated from ref. $(13,14), * 3$ from ref. (22,

23), and other values from ref. (11). PAG, pulmonary angiography.

\section{Results}

\section{Prospective case-registry}

There were 58 enrolled cases. In the 58 cases, 30 [51.7\%; 95\% confidence interval (CI), 39.2-64.1\%] were introduced before using diagnostic imaging techniques including lung scan, pulmonary angiography, CT, and MR angiography. APTE was found in $45(77.6 \%, 95 \% \mathrm{CI}: 65.3-86.4 \%)$ of all registry cases, and in $20(66.7 \%, 95 \%$ CI: $48.8-80.8 \%)$ before using diagnostic imaging techniques. As shown in Fig. 2, the probability of the presence of APTE determined by the participating doctor was related to the final diagnosis.

Compared with the absence of APTE, prolonged immobilization and cancer were found more often in presence of APTE (Table 2). As for symptoms and signs, tachycardia and unilateral leg swelling were found more often in the presence of APTE (Table 3). Inverted $\mathrm{T}$-wave in $\mathrm{V}_{1-3}$ was found more often and the level of D-dimer was higher in the presence of APE (Table 4). The level of D-dimer was higher than $0.5 \mu \mathrm{g} / \mathrm{ml}$ in all enrolled cases.

The areas of the receiver operating characteristics (ROC) curve in the heart rate and D-dimer level were $0.74(95 \%$ CI, 0.59-0.90; $\mathrm{p}=0.01)$ and $0.87(0.73-0.997 ; \mathrm{p}=0.0002)$, respectively (Fig. 3). 

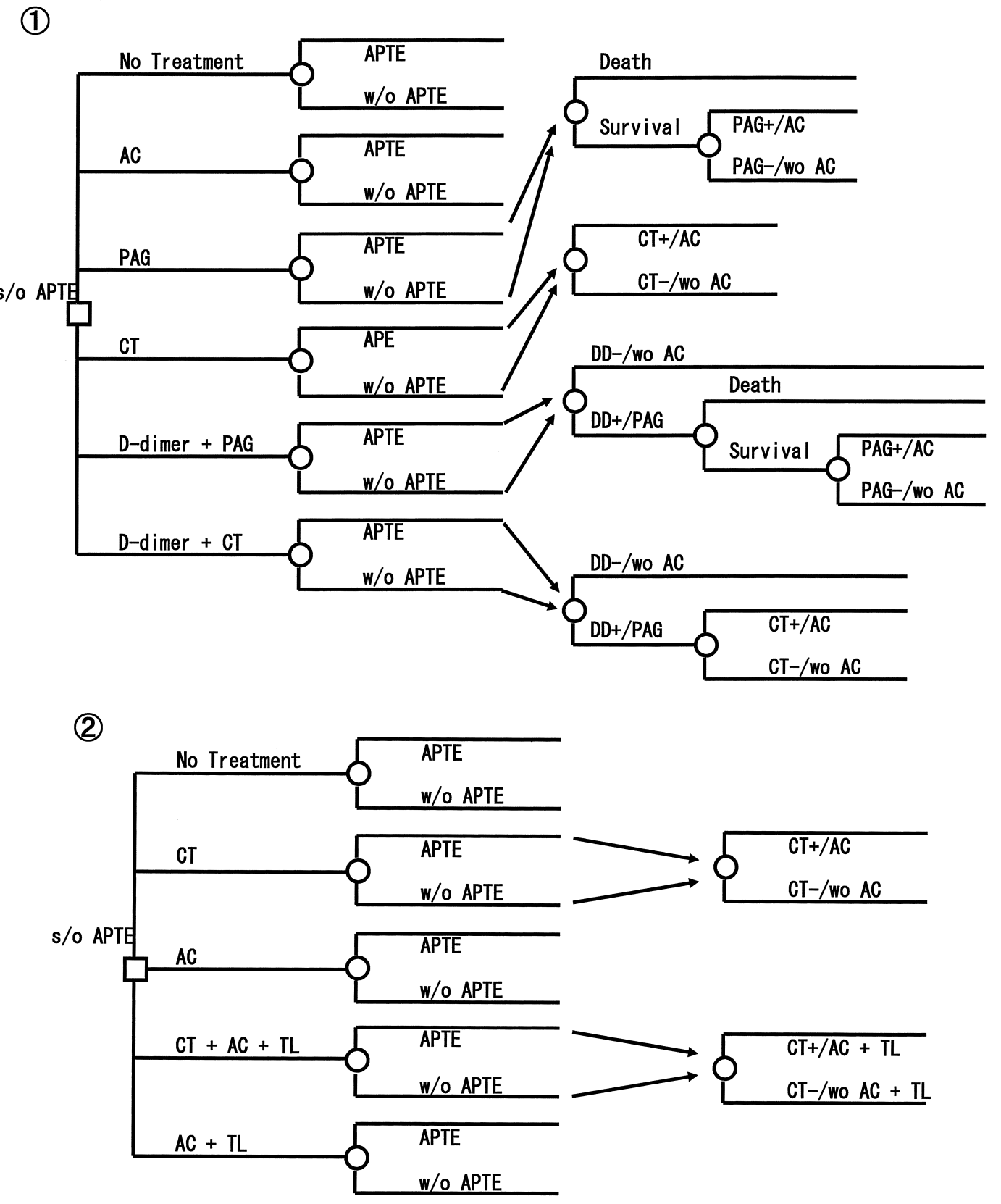

Figure 1. Decision tree for diagnosis and treatment of acute pulmonary thromboembolism (APTE). AC, anticoagulant; TL, thrombolysis; PAG, pulmonary angiography; CT, computed tomography; DD, D-dimer; s/o, suspected of; w/o, without; +, positive result; -, negative result; $\square$, decision node; $\bigcirc$, chance node.

\section{The examination tests of APTE}

The rate and the median value of the duration in getting the result on the day of ordering the examination test was $100 \%$ and 5 minutes for arterial blood gas analysis, $78.2 \%$ and 45 minutes for D-dimer, $100 \%$ and 15 minutes for trans-thoracic echocardiography, $54.5 \%$ and 1 hour for perfusion lung scan, $3.6 \%$ and 2 hours for ventilation lung scan, $85.5 \%$ and 1 hour for pulmonary angiography, $100 \%$ and 1 hour for CT, $36.4 \%$ and 1 hour for MR angiography, and $56.4 \%$ and 50 minutes for trans-esophageal echocardiography, respectively.

D-dimer was measured in $52.7 \%$ of the cases by latex ag- glutination assay, in $38.2 \%$ by latex immunoturbidimetric assay, in $7.3 \%$ by enzyme-linked immunosorbent assay, and $1.8 \%$ by other assays. Multi detector-row CT was $81.8 \%$ of CT.

\section{Decision analysis}

Fig. 4 is the result of the decision analysis for the decision tree shown in Fig. 1-11. Among the decision branches, the highest expected utility was anticoagulant over 0.51 in the pre-test probability, CT between 0.13 and 0.51 , D-dimer and CT between 0.007 and 0.13 , and no therapy below 0.007 .

Fig. 5 is the result of the decision analysis for the deci- 


\section{Presence of APTE}

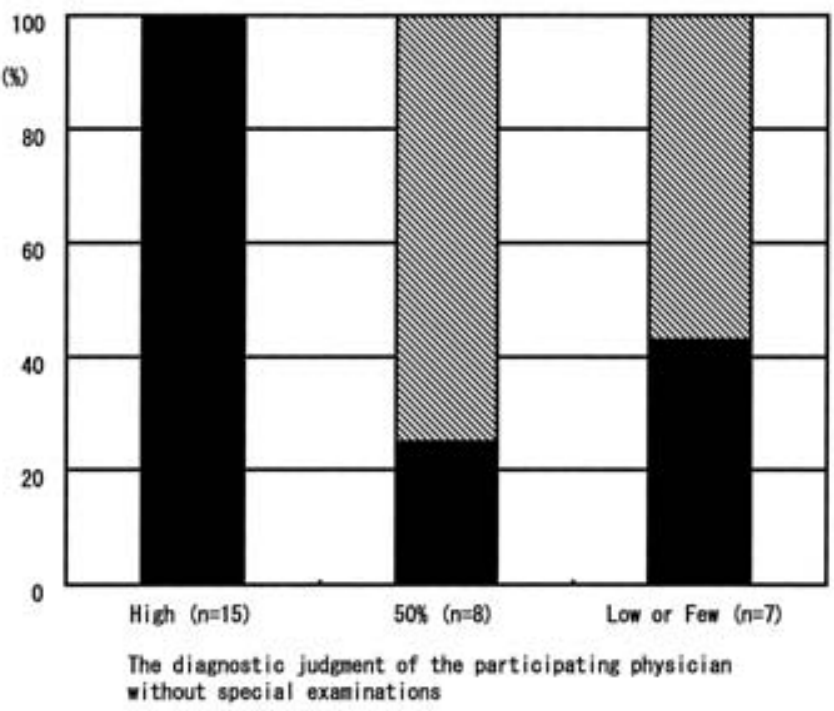

Figure 2. Relationship between the probability of the presence of acute pulmonary thromboembolism (APTE) determined by the participating doctor and the final diagnosis. Filled bars show APTE present and shaded bars show APTE absent. $\mathbf{p}=\mathbf{0 . 0 0 0 4}$ among rate of final APTE diagnosis at three levels (High, $50 \%$, and Low or Few) of diagnostic judgment of the participating physician without special imaging examinations.

Table 2. Patient Characteristics in the Presence and Absence of APTE

\begin{tabular}{lccc}
\hline Characteristics & APTE Present & APTE Absent & p-value \\
\hline Age $(\mathrm{y} . \mathrm{o}) *$ & $66(49-75.5 ; 45)$ & $54(36.5-75.5 ; 13)$ & 0.39 \\
Female & $26 / 45(57.8 \%)$ & $9 / 13(69.2 \%)$ & 0.53 \\
BMI $*$ & $25.1(23.3-28.1 ; 37)$ & $25.2(21.7-30.0 ; 13)$ & 0.69 \\
Prolonged immobilization & $23 / 45(51.1 \%)$ & $2 / 13(15.4 \%)$ & 0.03 \\
Recent major surgery & $18 / 45(40.0 \%)$ & $4 / 13(30.8 \%)$ & 0.75 \\
Recent fracture & $7 / 45(15.6 \%)$ & $4 / 13(30.8 \%)$ & 0.24 \\
History of DVT & $5 / 45(11.1 \%)$ & $1 / 13(7.7 \%)$ & 1.00 \\
Active cancer & $15 / 45(33.3 \%)$ & $0 / 13(0.0 \%)$ & 0.01 \\
Circumstance at onset & & & \\
Urination or defecation & $3 / 45(6.7 \%)$ & $0 / 13(0.0 \%)$ & 1.00 \\
Standing up or walking & $10 / 45(22.2 \%)$ & $1 / 13(7.7 \%)$ & 0.43 \\
\hline
\end{tabular}

APTE, acute pulmonary thromboembolism; BMI, body mass index; DVT, deep vein thrombosis. $*$ Median (25 percentile-75 percentile; number of cases).

sion tree shown in Fig. 1-(2), where mortalities from APTE itself without therapy, using anticoagulant, and using both anticoagulant and thrombolytic agent were variable. The combination of anticoagulant and thrombolytic agent is the best choice when the reduction of mortality by the combination is much greater than that by anticoagulant alone. And this is more likely to occur when the pre-test probability is higher.
Table 3. Symptoms and Signs in the Presence and Absence of APTE

\begin{tabular}{lccc}
\hline & APTE Present & APTE Absent & p-value \\
\hline Symptoms & & & \\
Dyspnea (sudden onset) & $24 / 44(54.5 \%)$ & $4 / 13(30.8 \%)$ & 0.21 \\
Dyspnea (gradual onset) & $17 / 44(38.6 \%)$ & $7 / 13(53.8 \%)$ & 0.36 \\
Chest pain (pleuritic) & $8 / 44(18.2 \%)$ & $4 / 13(30.8 \%)$ & 0.44 \\
Chest pain (substernal) & $6 / 45(13.3 \%)$ & $2 / 13(15.4 \%)$ & 1.00 \\
Syncope & $6 / 44(13.6 \%)$ & $0 / 13(0.0 \%)$ & 0.32 \\
Shock & $9 / 45(20.0 \%)$ & $0 / 13(0.0 \%)$ & 0.19 \\
Signs & & & \\
Pulse (beats/min) * & $100(84.5-110 ; 41)$ & $83.3(69.3-94.5 ; 12)$ & 0.01 \\
Respiratory rate $(/$ min) * & $24(19-28 ; 19)$ & $15.5(12-27.8 ; 8)$ & 0.20 \\
Systolic blood pressure (mmHg) * & $126(101-136.5 ; 45)$ & $124(108-132 ; 13)$ & 0.90 \\
Diastolic blood pressure (mmHg)* & $75(63-82 ; 40)$ & $70(63-86 ; 13)$ & 0.99 \\
Body temperature $\left(^{\circ} \mathrm{C}\right) *$ & $36.8(36.5-37.6 ; 33)$ & $36.8(36.2-37.0 ; 11)$ & 0.41 \\
Leg swelling (Unilateral) & $14 / 45(31.1 \%)$ & $0 / 13(0.0 \%)$ & 0.03 \\
Neck vein distention & $6 / 43(14.0 \%)$ & $1 / 13(7.7 \%)$ & 1.00 \\
Wheezing & $2 / 44(4.5 \%)$ & $2 / 13(15.4 \%)$ & 0.22 \\
\hline
\end{tabular}

APTE, acute pulmonary thromboembolism. * Median (25 percentile- 75 percentile; number of cases).

Table 4. Chest Roentgenogram, Electrocardiogram, Echocardiogram, and D-dimer Findings in the Presence and Absence of APTE

\begin{tabular}{lccc}
\hline & APTE Present & APTE Absent & p-value \\
\hline Chest roentgenogram & & & \\
Oligemia & $9 / 43(20.9 \%)$ & $3 / 13(23.1 \%)$ & 1.00 \\
PA dilatation & $11 / 43(25.6 \%)$ & $1 / 13(7.7 \%)$ & 0.26 \\
Infiltration & $2 / 43(4.7 \%)$ & $2 / 13(15.4 \%)$ & 0.23 \\
Electrocardiogram & & & \\
Inverted T-wave in $\mathrm{V}_{1}-\mathrm{V}_{3}$ & $19 / 40(47.5 \%)$ & $1 / 13(7.7 \%)$ & 0.02 \\
SiQIII or SiQmIIII & $16 / 40(40.0 \%)$ & $2 / 13(15.4 \%)$ & 0.18 \\
RBBB & $3 / 40(7.5 \%)$ & $0 / 0(0.0 \%)$ & 0.57 \\
Echocardiography & & & \\
Right ventricular dilatation & $20 / 36(55.6 \%)$ & $3 / 9(33.3 \%)$ & 0.28 \\
Right ventricular hypokinesis & $2 / 36(5.6 \%)$ & $0 / 9(0.0 \%)$ & 1.00 \\
D-dimer $(\mu \mathrm{g} / \mathrm{ml}) *$ & $19.8(7.6-33.1 ; 38)$ & $2.5(1.4-4.9 ; 12)$ & 0.0002 \\
\hline
\end{tabular}

APTE, acute pulmonary thromboembolism; RBBB, transient right bundle branch block. * Median (25 percentile- 75 percentile; number of cases).

\section{Discussion}

Diagnostic strategies of APTE have been discussed in various reports and Western guidelines (8-10). It then needs to be determined whether the Western approaches are applicable to the situation in Japan. The sensitivity and the specificity of examination tests are thought to be universal. Therefore, three unique points in Japan are as follows. The first is the way to elevate the efficiency of the pre-test probability before using imaging techniques specific to APTE in patients suspected of APTE. The second is the selection of procedures that can be employed rapidly and are available in most hospitals. And the last is to confirm the efficacy of the diagnostic and therapeutic procedures by decision analysis. 
Sensitivity

(True-positive Rate)

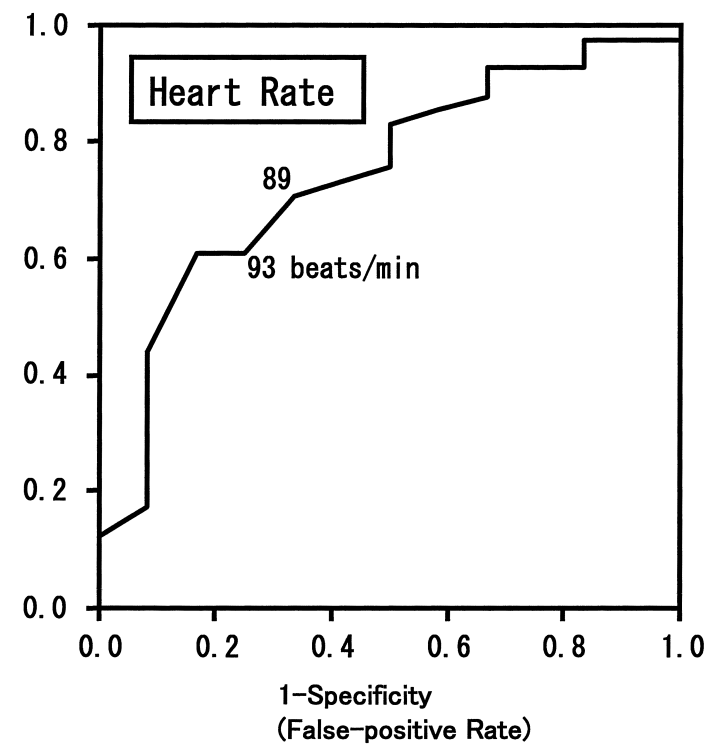

Sensitivity

(True-positive Rate)

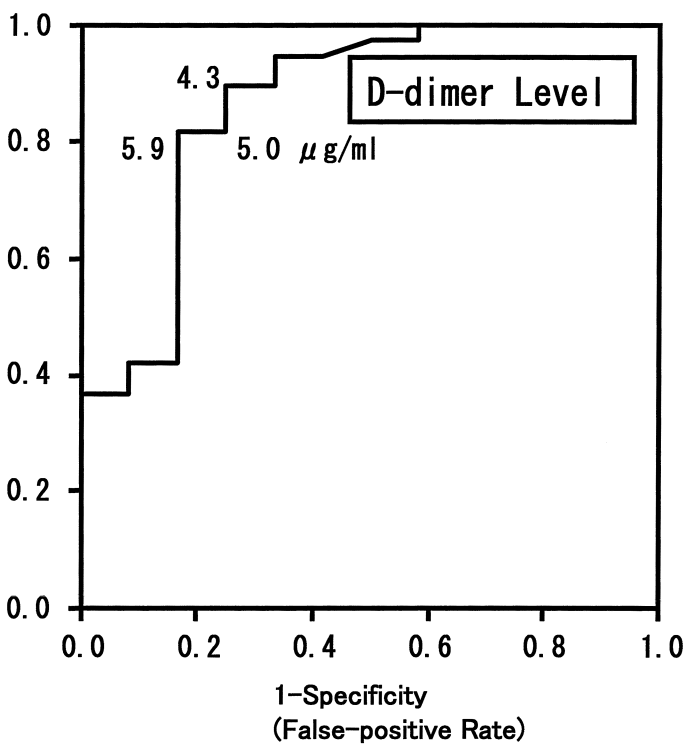

Figure 3. Receiver operating characteristics (ROC) curve in heart rate and D-dimer level.

\section{Before using imaging techniques specific to APTE}

The pre-test probability of APTE had already been high before using specific diagnostic imaging techniques. Our data suggested that some findings could be used to elevate further the pre-test probability.

APTE was present in $66.7 \%$ of cases introduced to a member of JaSPER before using specific imaging techniques. This means that the pre-test probability is high in Japan compared with that in previous reports in Western countries [ $40 \%$ of test group and $42 \%$ of validation group by Miniati et al (5), 33\% of test group and $41 \%$ of validation group by Hoellerich and Wigton (6), and $55 \%$ by Celi et al (7)]. There are two potential reasons. The first is that APTE had been suspected less frequently and only typical cases had been diagnosed in Japan. The second potential reason is that APTE patients had been effectively selected, which cannot be completely ruled out because all our cases were from selected hospitals, members of JaSPER.

Autopsy studies showed that the death rate from APTE in Japanese was similar to that in non-blacks non-whites in the United States $(15,16)$ which was $39 \%$ of that in white Americans (16). And in the United States clinical data also showed similar racial differences: clinical cases per population in non-black non-white Americans [7 cases/100,000 person-year (17)] were 20-29\% of white Americans $(17,18)$. On the other hand, APTE in Japan was diagnosed in as few as 2.8-3.2 cases $/ 100,000$ person-years $(1,3)$. These show that the difference in clinically diagnosed rates between Japan and the United States is far from the difference in rates found by autopsy between Japan and the United States, and imply that the diagnostic power is still low in Japan.

Analysis of the 58 enrolled cases revealed that there were significant differences in the rate of prolonged immobilization, active cancer, unilateral leg swelling, and negative Twave in $\mathrm{V}_{1-3}$, and the value of heart rate and $\mathrm{D}$-dimer between patients with APE and those without. These results suggested that patients of high probability for APTE were drawn from patients suspected of APTE.

Some problems were revealed from the present caseregistry. In spite of the fact that this was a prospective study and that the examination items were known ahead of time, basic vital signs, especially the respiratory rate, were not or could not be obtained in many cases. Vital signs are fundamental, and their importance should be recognized.

\section{Diagnostic procedure}

We showed that the diagnostic strategy for APTE made by the Japanese Circulation Society (19) was available in most hospitals in Japan, and that the central diagnostic procedures might be arterial blood gas analysis, D-dimer, CT, and pulmonary angiography.

It is important to make a diagnostic strategy based on examination tests the results of which can be obtained on the same day, because deaths from APTE occur mainly on the day of diagnosing APE and the day after (20). From this viewpoint, it is not practical for ventilation and perfusion lung scan to be used in the diagnostic strategy in Japan as in Western countries.

The examination results of CT, trans-thoracic echocardiography and arterial blood gas analysis were obtained on the day ordered in all hospitals. Those of pulmonary angiography were also available in a large number of hospitals. It is widely accepted that a low level of D-dimer is useful to exclude a diagnosis of APTE. But there were two problems of D-dimer in Japan. One was that the result of the D-dimer level was obtained on the day ordered in many hospitals but 


\section{Expected Utility}

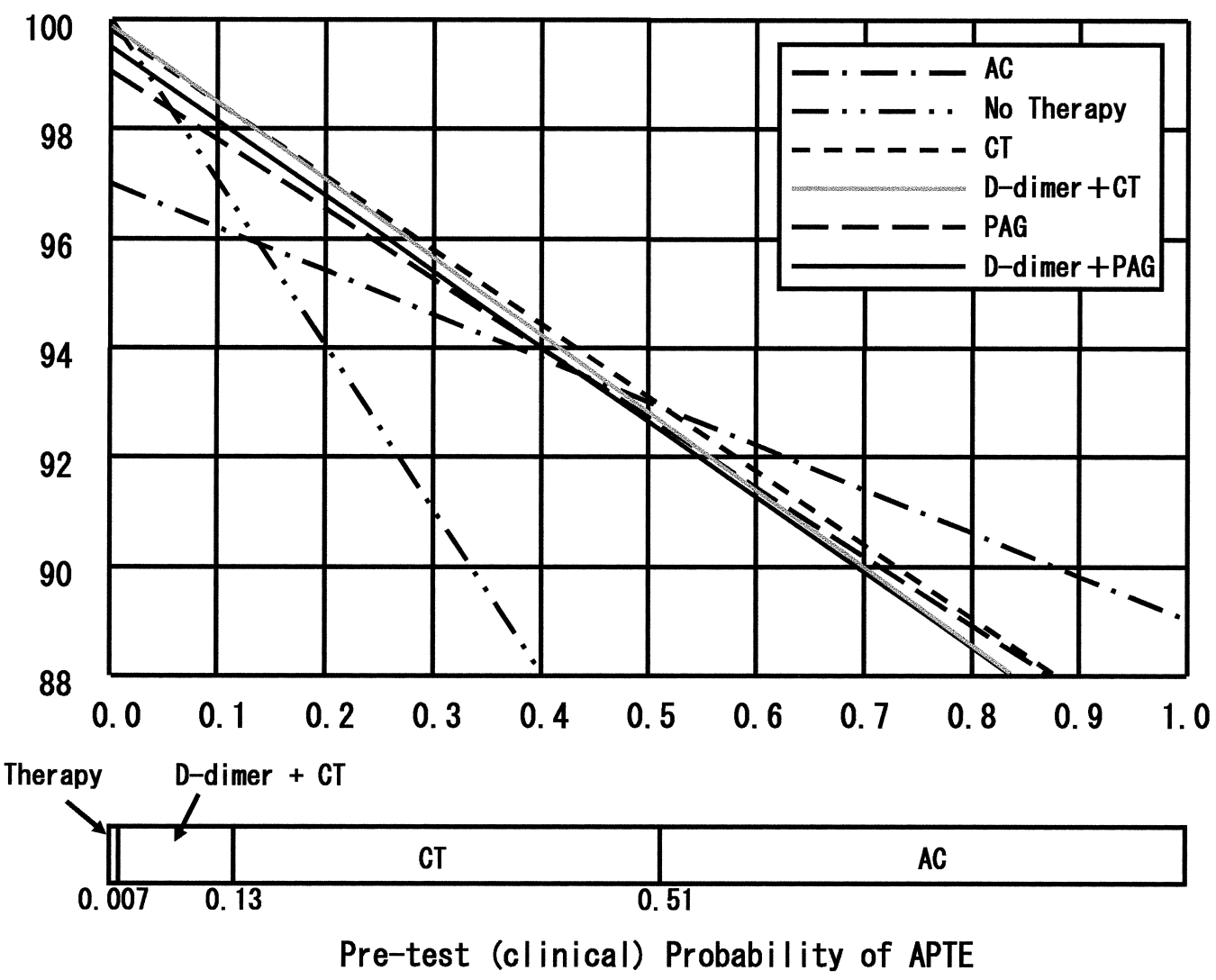

Figure 4. Pre-test probability of acute pulmonary thromboembolism (APTE) and expected utility. Expected utility was calculated using values in Table 1 and decision tree shown in Fig. 1- (1) . Lower bar indicates the highest ranking strategy at certain pre-test probabilities. AC, anticoagulant; CT, computed tomography; PAG, pulmonary angiography.

not in all hospitals. Another was the use of non-quantitative assays in over half of the hospitals. These can be improved with minimal investment. The results of perfusion lung scan and ventilation lung scan can be obtained on the day ordered in only $54.5 \%$ and $3.6 \%$ of the hospitals, respectively. This results from the necessity for careful handling of radioisotopes in Japan. The specificity of lung scan is inferior to those of CT, pulmonary angiography and MR angiography $(11,12,21)$. It is improbable that, in the future, lung scan will become the major diagnostic test for APTE in Japan, because lung scan cannot be used for a final diagnosis in most APTE suspected patients and involves some other difficulties as described above.

In Western countries, non-invasive tests are desirable and pulmonary angiography has been used as little as possible. But in Japan, invasive treatments including inferior vena cava filter and catheter therapy were performed aggressively and, in diagnosis, many physicians do not hesitate to select an invasive diagnostic tool such as pulmonary angiography.

In 2004, guidelines for a diagnostic strategy for APTE were published in Japan (Fig. 6) (19). Of course, pulmonary angiography cannot be carried out in all hospitals. Pulmonary angiography is only one choice of diagnostic procedures under the Japanese guideline. Accordingly, each insti- tute can select a diagnostic procedure suitable for the needs and actual condition of the patient. The present results on the examination tests for APTE show that the guidelines conform to the present clinical condition in Japan and can be carried out in practice.

\section{Decision analysis}

From the decision analysis, anticoagulant has to be chosen in patients with suspected APTE in Japan because the pre-test probability of APTE was over $60 \%$, when the use of thrombolysis was not needed for the differential diagnosis. Given that thrombolytic agents lower the mortality from APTE to a great degree compared with anticoagulant use alone, thrombolytic agents can be used theoretically without confirmation of the diagnosis.

Decision analysis comparing no treatment, heparin treatment, pulmonary angiography with and without D-dimer, and CT with and without D-dimer for patients with suspected APE indicated that CT and pulmonary angiography with and without D-dimer have higher utility than heparin when the pre-test probability was below about $50 \%$, and that the relation was reversed when the probability was over about $50 \%$. But there was little difference in the expected utility between CT and PAG with and without D-dimer. 
$A-B$

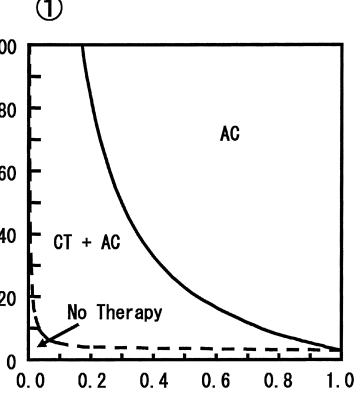

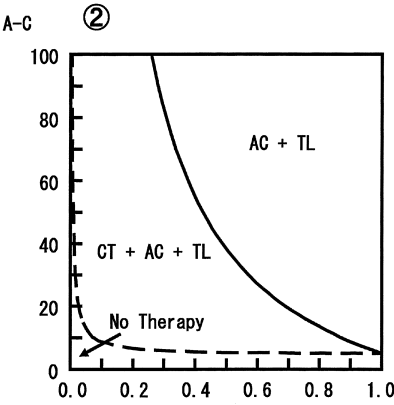

Pre-test (clinical) Probability of APTE
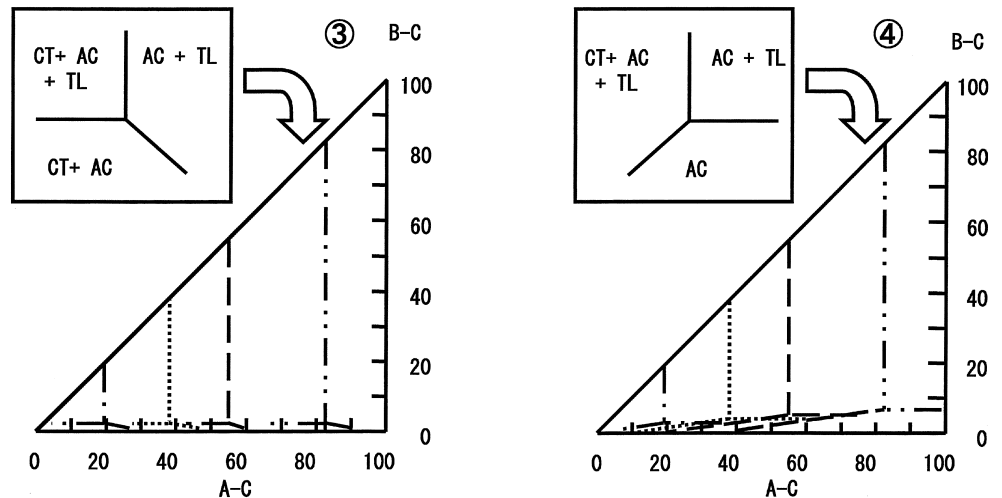

If no therapy in (1), then go to (2).

If $\mathrm{CT}+\mathrm{AC}$ in (1), then go to (3).

If $A C$ in (1), then go to (4).

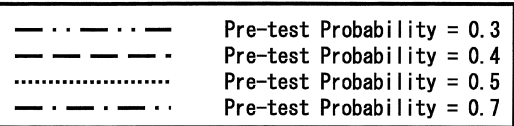

Figure 5. Conditions for obtaining maximum utility with the change in loss of utility by acute pulmonary thromboembolism (APTE) itself. We compared the following 5 conditions: 1) no treatment, 2) anticoagulant use when $\mathrm{CT}$ was positive, 3) anticoagulant use without $\mathrm{CT}, 4)$ combination use of anticoagulant and thrombolytic agent when CT was positive, and 5) combination use of anticoagulant and thrombolytic agent without CT. First, 1), 2) and 3) were compared using (1). The condition at the highest score of expected utility was obtained from the pre-test probability and value of $\mathrm{A}-\mathrm{B}$. If 1) was highest between 1), 2) and 3), 1), 4) and 5) were compared using (2). Otherwise, if 2) was highest, 2), 4) and 5) were compared using (3). An (A-C)-(B-C) plane was divided into three portions to obtain the maximum utility by the pre-test probability. The highest utility was under the condition of 2) at the lower portion of the plane, 4) at the left upper portion, and 5) at the rightward portion as shown in the sub-window of the figure. Otherwise, if 3 ) was highest, 3), 4) and 5) were compared using (4). An (A-C)-(B-C) plane was divided into three portions to obtain maximum utility by the pre-test probability. The highest utility was under the condition of 3 ) at the lower portion of the plane, 4) at the leftward portion, and 5) at the right upper portion as shown in the sub-window of the figure. The expected utility was calculated using the values in Table 1 and the decision tree shown in Fig. 3- (2), where the mortalities by APTE without treatment (A), treating by anticoagulant (B) and by a combination of anticoagulant and thrombolytic agents (C) were variable. We assumed $A>B>C$. Otherwise, each therapy is apparently useless. AC, anticoagulant; TL, thrombolysis; CT, computed tomography.

Consideration of the pre-test probability of APTE in Japan before using specific imaging techniques, heparin is superior to other choices. This analysis was based on data showing that the loss of utility for APTE itself is 30 without treatment and 8 when using anticoagulants, and in our next step these values were changed to assess the influence on the clinical decision.

If thrombolytic therapy did not improve the mortality from APTE, thrombolysis would have apparently lower util- ity than heparin because of the increase in major bleeding $(22,23)$. On the other hand, Fig. 5 indicates that, when the improvement of mortality from APTE itself is much greater by using thrombolytic agents and anticoagulant than by anticoagulant alone, the expected utility is high using thrombolytic agents and anticoagulant compared with that in other choices even if the pre-test probability is low. Bottiger et al suggested that thrombolysis with heparin might improve the mortality in patients with initial, unsuccessful treatment of 


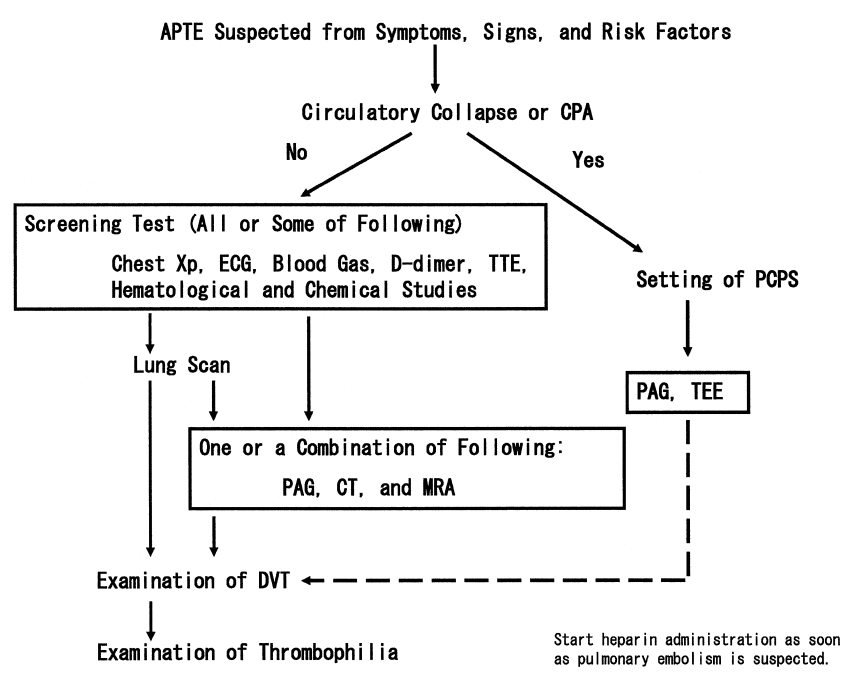

Figure 6. Diagnostic strategy for acute pulmonary thromboembolism (APTE). Heparin is administered when APTE is suspected. CPA, cardio-pulmonary arrest; Xp, roentgenogram; ECG, electrocardiography; TTE, trans-thoracic echocardiography; PAG, pulmonary angiography; CT, computed tomography; MRA, magnetic resonance angiography; TEE, trans-esophageal echocardiography; DVT, deep vein thromb: sis; PCPS, percutaneous cardio-pulmonary support. (From The Japanese Circulation Society. Guidelines for the diagnosis, treatment and prevention of pulmonary thromboembolism and deep vein thrombosis (JCS 2004). Circ J 2004; 68 (Suppl IV): 1079-1134 (in Japanese) with permission).

cardiopulmonary arrest caused by cardiac reasons including pulmonary embolism and myocardial infarction (24). Their findings appear to be in agreement with the situation described above.

Therapeutic strategy (Fig. 7) was also described in the guidelines (19), but we have no data by which we can assess the strategy objectively. We may be able to choose the therapy for APTE by decision analysis when mortalities both according to the severity of the disease and according to each type of therapy are obtained.

\section{Conclusion}

The pre-test probability of APTE was already high before using specific diagnostic imaging techniques in Japan. Therefore, anticoagulant was the best choice in patients with suspected APTE in Japan when we did not need to use thrombolysis nor to distinguish APTE from other diseases. Our results showed that the diagnostic strategy for APTE made by the Japanese Circulation Society was available in most hospitals in Japan.

\section{APPENDX 1}

The following centers participated in JaSPER: Asahi General Hospital, Asahi; Cardiopulmonary Division, Keio University School of Medicine, Tokyo; Cardiovascular Center,

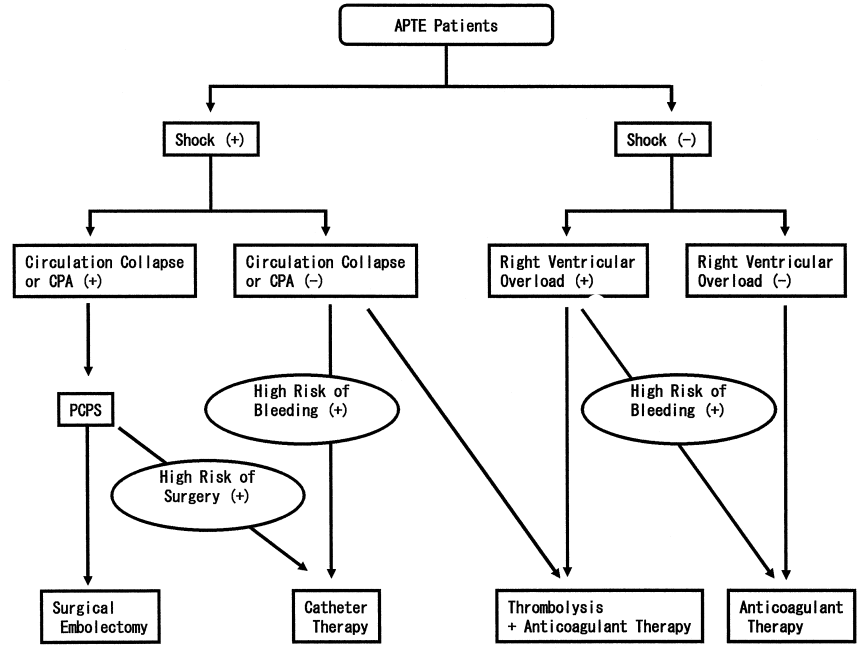

Figure 7. Therapeutic strategy for acute pulmonary thromboembolism (APTE). CPA, cardio-pulmonary arrest; PCPS, percutaneous cardio-pulmonary support. (From The Japanese Circulation Society. Guidelines for the diagnosis, treatment and prevention of pulmonary thromboembolism and deep vein thrombosis (JCS 2004). Circ J 2004; 68 (Suppl IV): 1079-1134 (in Japanese) with permission).

Fukushima Daiichi Hospital, Fukushima; Cardiovascular Center, Toho University Sakura Hospital, Sakura; Cardiovascular Center, Yokohama City University Medical Center, Yokohama; Department of Anesthesiology and Critical Care Medicine, Jichi Medical School, Tochigi; Department of Anesthesiology and Critical Care Medicine, Jichi Medical University Omiya Medical Center, Omiya; Department of Anesthesiology, Kitasato University School of Medicine, Sagamihara; Department of Anesthesiology, Nara Medical University, Kashihara; Department of Cardiology and Pneumology, Dokkyo University School of Medicine, Mibu; Department of Cardiology, Kawasaki Medical School, Kurashiki; Department of Cardiology, Mie University Graduate School of Medicine, Tsu; Department of Cardiology, The Jikei University School of Medicine, Tokyo; Department of Cardiology, Tokai University School of Medicine, Isehara; Department of Cardiology, Tokyo Medical University Kasumigaura Hospital, Ibaraki; Department of Cardiothoracic Surgery, University of Tokyo Graduate School of Medicine, Tokyo; Department of Cardiovascular and Renal Medicine, Saga University Faculty of Medicine, Saga; Department of Cardiovascular Medicine, Kyoto University Graduate School of Medicine, Kyoto; Department of Cardiovascular Medicine, Tohoku University Graduate School of Medicine, Sendai; Department of Cardiovascular Medicine, University of Tokyo Graduate School of Medicine, Tokyo; Department of Cardiovascular Surgery, Graduate School of Medical Sciences, Kumamoto University, Kumamoto; Department of Cardiovascular Surgery, Kanazawa Medical University, Ishikawa; Department of Cardiovascular Surgery, Kobe University Graduate School of Medicine, Kobe; Department of Cardiovascular Surgery, National Cardiovascular Center, Osaka; 
Department of Cardiovascular Physiology and Medicine Hiroshima University Graduate School, Hiroshima; Department of Clinical Laboratory Medicine, Kyoto University Graduate School of Medicine, Kyoto; Department of Diagnostic Radiology, Keio University School of Medicine, Tokyo; Department of Emergency and Critical Care Medicine, Hyogo College of Medicine, Nishinomiya; Department of Emergency and Critical Care Medicine, Sapporo Medical University School of Medicine, Sapporo; Department of Emergency Critical Care Medicine, St. Marianna University School of Medicine, Kawasaki; Department of Internal Medicine and Cardiology, Osaka City University Graduate School of Medicine, Osaka; Department of Internal Medicine, National Cardiovascular Center, Osaka; Department of Internal Medicine, Sumida Shuko-en Facility, Tokyo; Department of Laboratory Medicine, Mie University School of Medicine, Tsu; Department of Life Science, Okayama University of Science, Okayama; Department of Medicine II, Iwate Medical University, Morioka; Department of Medicine, Tokai University Tokyo Hospital, Tokyo; Department of Molecular Medicine, Osaka University Graduate School of Medicine, Osaka; Department of Obstetrics and Gynecology, Hamamatsu University School of Medicine, Hamamatsu; Department of Obstetrics and Gynecology, Nara Medical University, Kashihara; Department of Obstetrics and Gynecology, Saitama Medical Center, Saitama Medical School, Saitama; Department of Orthopedic Surgery, Mie University School of Medicine, Tsu; Department of Pulmonary Medicine, Fukushima Medical University School of Medicine, Fukushima; Department of Radiology, Graduate School of Medical Science, Kyoto Prefectural University of Medicine, Kyoto; Department of Radiology, Mie University School of Medicine, Tsu; Department of Radiology, Nippon Medical School, Tokyo; Department of Radiology, St. Marianna University School of Medicine, Kawasaki; Department of Respiratory Medicine, Saitama Medical School, Saitama; Department of Respiratory Medicine, Showa University Fujigaoka Hospital, Yokohama; Department of Respiratory, Medicine, Graduate School of Medicine, Hokkaido University, Sapporo; Department of Respirology, Chiba University Graduate School of Medicine, Chiba; Department of Surgery and Clinical Oncology, Osaka University Graduate School of Medicine, Osaka; Department of Surgery I, Hirosaki University School of Medicine, Hirosaki; Department of Surgery, Jichi Medical University, Tochigi; Department of Surgery, Tokyo Medical College, Tokyo; Departments of Cardiology, Gifu University Graduate School of Medicine, Gifu; Division of Cardiology, Fujita Health University School of Medicine, Toyoake; Division of Cardiology, St. Marianna University School of Medicine, Kawasaki; Division of Cardiovascular and Respiratory Medicine, Kobe University Graduate School of Medicine, Kobe; Division of Cardiovascular Surgery, Fujita Health University, Toyoake; Division of Intensive and Coronary Care Unit, Nippon Medical School, Tokyo; Division of Interventional Cardiology, Toho University Omori Medical Center, Tokyo; Division of Organ Re- generation Surgery, Tottori University Faculty of Medicine, Yonago; Division of Respiratory Medicine, Toho University Omori Medical Center, Tokyo; Division of Thoracic and Cardiovascular Surgery, Niigata University Graduate School of Medical and Dental Sciences, Niigata; First Department of Internal Medicine, Nippon Medical School, Tokyo; First Department of Internal Medicine, Yamagata University School of Medicine, Yamagata; Gifu Prefectural Tajimi Hospital, Tajimi; Handa City Hospital, Handa; Hiroshima City Hospital, Hiroshima; Ichinomiya City Hospital, Ichinomiya; JR Tokyo General Hospital, Tokyo; Kanagawa Cardiovascular and Respiratory Center, Yokohama; Kobe City General Hospital, Kobe; Koebaru Chuo Hospital, Nagasaki; Kokura Memorial Hospital, Kitakyushu; Komaki City Hospital, Komaki; Matsuda Hospital, Kurashiki; Mie Prefectural General Medical Center, Yokkaichi; Musashino Red Cross Hospital, Musashino; Nagoyacity, Jouhoku Municipal Hospital; Narita Red Cross Hospital, Narita; National Cancer Center Hospital; National Hospital Organization Chiba Medical Center, Chiba; National Hospital Organization Zentsuji National Hospital, Zentsuji; Nishinomiya Municipal Central Hospital, Nishinomiya; Ofuna Chuo General Hospital, Kamakura; Ogaki Municipal Hospital, Ogaki; Saiseikai Matsusaka General Hospital; Saiseikai Yokohama-shi Nambu Hospital, Yokohama; Saitama Cardiovascular and Respiratory Center, Saitama; Sasebo City General Hospital, Sasebo; Sawada Hospital, Gifu; Second Department of Internal Medicine, Faculty of Medicine, University of the Ryukyus, Nishihara; Second Department of Internal Medicine, Kyorin University School of Medicine, Mitaka; Second Department of Internal Medicine, Nagasaki University School of Medicine, Nagasaki; Second Department of Internal Medicine, Nara Medical University, Kashihara; Second Department of Surgery, Ehime University School of Medicine, Toon; Seirei Mikatahara General Hospital, Shizuoka; Shinshu University School of Health Sciences, Matsumoto; Showa General Hospital, Kodaira; Suzuka General Hospital, Suzuka; Takarazuka Daiichi Hospital, Takarazuka; Tamana Central Hospital, Tamana; The Cardiovascular Division, Jichi Medical University Omiya Medical Center, Omiya; The Department of Cardiology, Kinki University School of Medicine, Osakasayama; The First Department of Medicine, Asahikawa Medical College, Asahikawa; The Secomd Department of Internal Medicine, Hirosaki University School of Medicine, Hirosaki; Tokyo Medical Examiner's Office, Tokyo; Tokyo Metropolitan Hiroo Hospital, Tokyo; Tokyo Metropolitan Otsuka Hospital, Tokyo; Tokyo Teishin Hospital, Tokyo; Toranomon Hospital, Tokyo; Yamada Red Cross Hospital, Ise; Yamato City Hospital, Yamato.

\section{APPENDIX 2}

Decision analysis has been used for the application of evidence-based medicine to practical medicine.

Calculation of the Post-probability of APTE (5)

Post-probability (ranging between 0 and 1) of APTE can 
be calculated using Bayes' theorem of conditional probability. The post-probability of APTE, conditioned by a positive diagnostic result, is described by the following equation:

$$
\frac{\text { sensitivity } \times \text { preP }}{(\text { sensitivity } \times \text { preP })+\{(1-\text { specificity }) \times(1-\text { preP })\}}
$$

where preP is the pre-test probability. Then, the postprobability of APTE, conditioned by a negative diagnostic result, is described by the following equation:

$$
\frac{(1-\text { sensitivity }) \times \text { preP }}{\{(1-\text { sensitivity }) \times \text { preP }\}+\{\text { specificity } \times(1-\text { preP })\}}
$$

Calculation of Expected Utility (EU)

Values of EU range from 0 (dead) to 100 (alive without morbidity). We dealt with 10 events of morbidity as one event of mortality as in Perrier's report (11). The EU value at the right border of each decision branch in Fig. 1 was calculated by the following equation:

$$
100-\text { Mortal ity }(\%)-\frac{\text { Morbidity }(\%)}{10}
$$

where Mortality and Morbidity include those due to APE itself, the examination test, and treatment. For example, the EU at the branch in non-treatment cases with APTE is 70, because the mortality is $30 \%$ in this situation (Table 1), and that in cases without APE is 100. Then, EU at each division just rightward of the decision node (see Fig. 1) was calculated by the following equation: $\sum_{i} P_{i} \times E U_{i}$, where $P_{i}$ is the probability (ranging between 0 and 1 ) and $\mathrm{EU}_{\mathrm{i}}$ (ranging between 0 and 100) is EU value at condition i. When the preprobability is $66.7 \%$, the EU of non-treatment is $0.667 \times 70+$ $(1-0.667) \times 100$. At the decision branches including the examination tests, the post-probability is used according to the result of the examination instead of the pre-test probability.

The best decision is defined as having the highest EU at division just rightward of the decision node among all decision branches.

\section{References}

1. Kumasaka N, Sakuma M, Shirato K. Incidence of pulmonary thromboembolism in Japan. Jpn Circ J 63: 439-441, 1999.

2. Sakuma M, Konno Y, Shirato K. Increasing mortality from pulmonary embolism in Japan, 1951-2000. Circ J 66: 1144-1149, 2002.

3. Kitamukai O, Sakuma M, Takahashi T, Kagaya Y, Watanabe J, Shirato K. Incidence and characteristics of pulmonary thromboembolism in Japan 2000. Intern Med 42: 1090-1094, 2003.

4. Sakuma M, Okada O, Nakamura M, et al. Recent developments in diagnostic imaging techniques and management for acute pulmonary embolism: Multicenter registry by Japanese Society of Pulmonary Embolism Research. Intern Med 42: 470-476, 2003.

5. Miniati M, Prediletto R, Formichi B, et al. Accuracy of clinical assessment in the diagnosis of pulmonary embolism. Am J Respir Crit Care Med 159: 864-871, 1999.

6. Hoellerich VL, Wigton RS. Diagnosing pulmonary embolism: Using clinical findings. Arch Intern Med 146: 1699-1704, 1986.

7. Celi A, Palla A, Petruzzelli S, et al. Prospective study of a standardized questionnaire to improve clinical estimate of pulmonary embolism. Chest 95: 332-337, 1989.

8. Hirsh J, Hoak J. Management of deep vein thrombosis and pulmonary embolism: A statement for healthcare professionals. Council on Thrombosis (in consultation with the Council on Cardiovascular Radiology), American Heart Association. Circulation 93: 22122245,1996

9. American Thoracic Society. The diagnostic approach to acute venous thromboembolism: Clinical practice guideline. Am J Respir Crit Care Med 160: 1043-1066, 1999.

10. Task Force on Pulmonary Embolism, European Society of Cardiology. Task force report: Guidelines on diagnosis and management of acute pulmonary embolism. Eur Heart J 21: 1301-1336, 2000.

11. Perrire A, Bounameaux H, Morabia A, et al. Contribution of Ddimer plasma measurement and lower-limb venous ultrasound to the diagnosis of pulmonary embolism: A decision analysis model. Am Heart J 127: 624-635, 1994.

12. Kline JA, Johns KL, Colucciello SA, Israel EG. New diagnostic tests for pulmonary embolism. Ann Emerg Med 35: 168-180, 2000.

13. Katayama H, Yamaguchi K, Kozuka T, Takashima T, Seez P, Ma- tsuura K. Adverse reactions to ionic and nonionic contrast media: A report from the Japanese Committee on the Safety of Contrast Media. Radiology 175: 621-628, 1990.

14. Munechika H, Hiramatsu $Y$, Kudo $S$, et al. A prospective survey of delayed adverse reactions to iohexol in urography and computed tomography. Eur Radiol 12: 185-194, 2003.

15. Sakuma M, Konno Y, Shirato K. Increasing mortality from pulmonary embolism in Japan, 1951-2000. Circ J 66: 1144-1149, 2002.

16. Lilienfeld DE. Decreasing mortality from pulmonary embolism in the United States, 1979-1996. Int J Epidemiol 29: 465-469, 2000.

17. Stein PD, Kayali F, Olson R, Milford CE. Pulmonary thromboembolism in Asians/Pacific islanders in the United States: Analysis of data from the National Hospital Discharge Survey and the United States Bureau of the Census. Am J Med 116: 435-442, 2004.

18. Klatsky AL, Armstrong MA, Poggi J. Risk of pulmonary embolism and/or deep venous thrombosis in Asian-Americans. Am J Cardiol 85: 1334-1337, 2000.

19. The Japanese Circulation Society. Guidelines for the diagnosis, treatment and prevention of pulmonary thromboembolism and deep vein thrombosis (JCS 2004). Circ J 68 (Suppl IV): 10791134, 2004 (in Japanese).

20. Sakuma M, Nakamura M, Nakanishi N, et al. Inferior vena cava filter is a new additional therapeutic option to reduce mortality from acute pulmonary embolism. Circ J 68: 816-821, 2004.

21. The PIOPED Investigators. Value of the ventilation/perfusion scan in acute pulmonary embolism: results of the Prospective Investigation of Pulmonary Embolism Diagnosis (PIOPED). JAMA 263: 2753-2759, 1990.

22. Thabut G, Thabut D, Myers RP, et al. Thrombolytic therapy of pulmonary embolism: A meta-analysis. J Am Coll Cardiol 40: 1660-1667, 2002.

23. Agnelli G, Becattini C, Kirschstein T. Thrombolysis vs heparin in the treatment of pulmonary embolism: A clinical outcome-based meta-analysis. Arch Intern Med 162: 2537-2541, 2002.

24. Bottiger BW, Bode C, Kern S, et al. Efficacy and safety of thrombolytic therapy after initially unsuccessful cardiopulmonary resuscitation: A prospective clinical trial. Lancet 3557: 1583-1585, 2001.

(C) 2006 The Japanese Society of Internal Medicine http://www.naika.or.jp/imindex.html 